\title{
Response to the Comments on Rimassa et al.: "Systemic Treatment Options in Hepatocellular Carcinoma"
}

\author{
Lorenza Rimassa $^{a} \quad$ Tiziana Pressiani $^{a} \quad$ Philippe Merle ${ }^{b}$ \\ ${ }^{a}$ Medical Oncology and Hematology Unit, Humanitas Cancer Center, Humanitas Clinical and

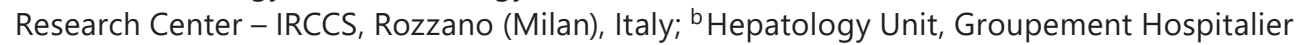 \\ Lyon Nord, Lyon, France
}

Dear Editor,

We appreciate Chen et al.'s [1] comments on our review on systemic treatment options for hepatocellular carcinoma (HCC) [2]. Chen and colleagues discuss three important aspects. First, the lack of predictive biomarkers for currently used targeted agents, which is a limitation for patient selection, and the need to identify such biomarkers. Chen et al. specifically mention MET as a potential predictive biomarker, but two phase 3 trials testing tivantinib (a MET inhibitor) and selecting patients according to MET expression failed to meet their primary endpoints [3,4]. The only positive phase 3 trial in a biomarker-selected population with HCC is the REACH-2 trial of ramucirumab in patients with high baseline alpha-fetoprotein levels [5]. Although we fully agree with Chen et al. that the lack of predictive biomarkers in HCC represents a highly unmet clinical need, at present we should base our clinical decisions on the data we have, and specifically on the results of clinical trials and on patient clinical characteristics. Further data on predictive biomarkers are certainly warranted.

Second, the potential role of systemic cytotoxic chemotherapy in combination with targeted agents such as sorafenib. No robust data demonstrated the efficacy and safety of chemotherapy in the treatment of patients with advanced HCC. The trial of FOLFOX4 versus doxorubicin [6] mentioned by Chen and colleagues compared two chemotherapy regimens but does not have a control arm with a standard sorafenib treatment; therefore, nothing can be concluded about the role of the FOLFOX4 regimen compared to targeted agents. With regard to the combination of chemotherapy and targeted agents, the CALGB 80802 phase 3 trial (testing the combination of sorafenib and doxorubicin versus sorafenib monotherapy) failed to show an improvement in survival in the face of added toxicity with the combination [7]. The phase 2 randomized PRODIGE 10 trial compared sorafenib alone versus sorafenib plus GEMOX (gemcitabine and oxaliplatin) and found limited progression-free survival benefit [8]. In the phase 3 RELIVE trial, following failure of sorafenib treatment, no improvement in overall survival was observed in patients treated with doxorubicin-loaded nanoparticles [9]. Given that now we have 5 drugs with positive phase 3 data (sorafenib, lenvatinib, regorafenib, cabozantinib, and ramucirumab [2]), we should base our clinical decisions on these data.

Third, the importance of considering patient-centered outcomes such as quality of life in the treatment decision making. We fully agree with Chen and colleagues that since we now have several active drugs available, we need to make our clinical decisions based not only on efficacy but on safety and quality of life data as well. Of note, the phase 3 trials of cabozantinib and ramucirumab showed an improvement in quality of life associated to the treatment compared to placebo [2]. 
In conclusion, the landscape of systemic treatment for advanced HCC is rapidly evolving, new targeted agents demonstrated their efficacy and tolerability in phase 3 trials, and checkpoint inhibitors results are more controversial, but combination phase 3 trials are ongoing; therefore, we have new therapeutic options for our patients and we should rely on the most robust data available.

\section{Acknowledgement}

Editorial/medical writing support provided by Susan M. Kaup, PhD, of inScience Communications, Springer Healthcare (Philadelphia, PA, USA), was funded by Exelixis, Inc.

\section{Statement of Ethics}

The authors have no ethical conflicts to disclose.

\section{Disclosure Statement}

Lorenza Rimassa declares advisory/consulting for Arqule, Bayer, Celgene, Eli Lilly, Eisai, Exelixis, Incyte, Ipsen, Sirtex Medical, and Roche, and speaker activities for AstraZeneca, AbbVie, and Gilead. Tiziana Pressiani has no conflicts of interest to declare. Philippe Merle declares advisory/consulting for Bayer, Bristol-Myers Squibb, Eli Lilly, Exelixis, Ipsen, Genosciences, Merck, Nanobiotix, Onxeo, and Roche.

\section{Funding Sources}

Funding for medical writing support was provided by Exelixis, Inc.

\section{Author Contributions}

All authors performed the writing and review and approved the response letter.

\section{References}

1 Chen M, Hu J, Cao J, Cai X. Comprehensive consideration before the decision-making of the systemic treatment in patients with advanced hepatocellular carcinoma - Letter. ((LIC 502775, wird zusammen publiziert im 1. Heft 2020) )

2 Rimassa L, Pressiani T, Merle P. Systemic treatment options in hepatocellular carcinoma. Liver Cancer 2019. https://doi: https://doi.org/10.1159/000499765.

3 Rimassa L, Assenat E, Peck-Radosavljevic M, Pracht M, Zagonel V, Mathurin P, et al. Tivantinib for second-line treatment of MET-high, advanced hepatocellular carcinoma (METIV-HCC): a final analysis of a phase 3, randomised, placebo-controlled study. Lancet Oncol. 2018 May;19(5):682-93.

4 Khobayashi S, Ueshima K, Moriguchi M, Takayama T, Izumi N, Yoshiji H, et al. JET-HCC: A phase 3 randomized, double-blind, placebo-controlled study of tivantinib as a second-line therapy in patients with c-Met high hepatocellular carcinoma. Ann Oncol. 2017;28(5 suppl):210.

5 Zhu AX, Kang YK, Yen CJ, Finn RS, Galle PR, Llovet JM, et al.; REACH-2 study investigators. Ramucirumab after sorafenib in patients with advanced hepatocellular carcinoma and increased $\alpha$-fetoprotein concentrations (REACH-2): a randomised, double-blind, placebo-controlled, phase 3 trial. Lancet Oncol. 2019 Feb;20(2):282-96.

6 Qin S, Bai Y, Lim HY, Thongprasert S, Chao Y, Fan J, et al. Randomized, multicenter, open-label study of oxaliplatin plus fluorouracil/leucovorin versus doxorubicin as palliative chemotherapy in patients with advanced hepatocellular carcinoma from Asia. J Clin Oncol. 2013 Oct;31(28):3501-8.

7 Abou-Alfa GK, Niedzwieski D, Knox JJ, Kaubisch A, Posey J, Tan BR, et al. Phase III randomized study of sorafenib plus doxorubicin versus sorafenib in patients with advanced hepatocellular carcinoma (HCC): CALGB 80802 (Alliance). J Clin Oncol. 2016 Feb;34(4 suppl):192.

8 Assenat E, Pageaux GP, Thézenas S, Peron JM, Bécouarn Y, Seitz JF, et al. Sorafenib alone vs. sorafenib plus GEMOX as 1st-line treatment for advanced HCC: the phase II randomised PRODIGE 10 trial. Br J Cancer. 2019 Apr;120(9):896-902.

9 Merle P, Blanc JF, Phelip JM, Pelletier G, Bronowicki JP, Touchefeu Y, et al.; RELIVE Investigators. Doxorubicinloaded nanoparticles for patients with advanced hepatocellular carcinoma after sorafenib treatment failure (RELIVE): a phase 3 randomised controlled trial. Lancet Gastroenterol Hepatol. 2019 Jun;4(6):454-65. 